\title{
Hypersurfaces with flat centroaffine metric and equations of associativity
}

\author{
Ferapontov E.V. \\ Department of Mathematical Sciences \\ Loughborough University \\ Loughborough, Leicestershire LE11 3TU \\ United Kingdom \\ e-mail: E.V.Ferapontov@lboro.ac.uk
}

\begin{abstract}
It is demonstrated that hypersurfaces $M^{n} \subset A^{n+1}$ with a flat centroaffine metric are governed by a system of nonlinear PDEs known as the equations of associativity of 2-dimensional topological field theory. In the case of surfaces $M^{2} \subset A^{3}$ this system reduces to a single third order PDE,

$$
f_{x x x} f_{y y y}-f_{x x y} f_{x y y}=1,
$$

where $x$ and $y$ are the asymptotic coordinates on $M^{2}$.
\end{abstract}

Keywords: centroaffine geometry, equations of associativity.

Mathematical Subject Classification: 53A40, 53A20, 35Q58, 35L60. 


\section{Introduction}

Centroaffine geometry studies the properties of hypersurfaces $M^{n} \subset A^{n+1}$ which are invariant under the action of the general linear group $G L(n+1)$. Since this action preserves the origin (translations are not allowed), one can choose the position vector $\mathbf{r}$ of a hypersurface as its centroaffine normal. With this normalization, one readily introduces the main centroaffine invariants: the centroaffine metric, the centroaffine cubic form and the Chebyshev covector (see sect. 1; in our presentation we follow the monograph [23], see also [22]). These invariants satisfy the compatibility conditions known as the centroaffine Gauss-Codazzi equations. It is well-known that the vanishing of the centroaffine cubic form characterises central hyperquadrics, while the vanishing of the Chebyshev covector is characteristic for proper affine hyperspheres. In this article we consider the third natural class of centroaffine hypersurfaces characterized by the flatness of the centroaffine metric.

The main observation is that, in the flat coordinates of the centroaffine metric, the corresponding Gauss-Codazzi equations reduce to a system of nonlinear PDEs known as the equations of associativity of two-dimensional topological field theory [6] (to be precise, WDVV equations without the quasihomogeneity condition). This is explained in sect. 3. For surfaces $M^{2} \subset A^{3}$ these equations reduce to a single PDE of the Monge-Ampere type,

$$
f_{x x x} f_{y y y}-f_{x x y} f_{x y y}=1,
$$

where $x$ and $y$ are the asymptotic coordinates on $M^{2}$.

The geometry of surfaces $M^{2} \subset A^{3}$ with a flat centroaffine metric is discussed in sect. 4 and 5 where, in particular, we establish some geometric properties of characteristics of the above PDE.

The review [6] provides an abundance of exact solutions of the associativity equations. Some of them are listed in sect. 5 and 6 . The centroaffine geometry of the corresponding surfaces is currently under investigation.

In sect. 7 a simple loop-group formulation of the linear system governing surfaces with a flat centroaffine metric is proposed which is due to A. Bobenko.

Since the centroaffine metric is proportional to the second fundamental form, hypersurfaces with a flat centroaffine metric (and projective transforms thereof) form a subclass of projective hypersurfaces with conformally flat second fundamental form which was investigated in detail in [2]. Another interesting class of projective surfaces $M^{2} \subset P^{3}$ (containing projective transforms of surfaces with a flat centroaffine metric as a proper subclass), is characterised by the existence of a one-parameter family of asymptotic deformations which induce constant rescalings of the projective metric and the Darboux cubic form. These generalizations are discussed in sect. 8 .

\section{Hypersurfaces in centroaffine geometry}

The position vector $\mathbf{r}=\left(r^{0}, r^{1}, \ldots, r^{n}\right)$ of a hypersurface $M^{n}$ in centroaffine geometry satisfies the linear system

$$
\mathbf{r}_{i j}=\tilde{\Gamma}_{i j}^{k} \mathbf{r}_{k}+g_{i j} \mathbf{r}, \quad i, j=1, \ldots, n
$$


which is required to be compatible of the rank $n+1$ (here $\mathbf{r}_{i}=\partial \mathbf{r} / \partial u^{i}, \mathbf{r}_{i j}=\partial \mathbf{r} / \partial u^{i} \partial u^{j}$ where $u^{1}, \ldots, u^{n}$ are local coordinates on $\left.M^{n}\right)$. Under reparametrizations of $M^{n}$, the objects $g_{i j}$ and $\tilde{\Gamma}_{i j}^{k}$ transform as the components of a pseudo-Riemannian metric (assumed nondegenerate) and Christoffel's symbols of an affine connection (called the affine connection of the first kind; we emphasize that it is not the Levi-Civita connection of $g_{i j}$ ). The conformal class of $g_{i j}$ is nothing but the second fundamental form of $M^{n}$. The compatibility conditions of system (11) are of the form [23

$$
\begin{gathered}
\tilde{\nabla}_{k} g_{i j}=\tilde{\nabla}_{j} g_{i k} \\
\tilde{R}_{i j k}^{s}=g_{i k} \delta_{j}^{s}-g_{i j} \delta_{k}^{s}
\end{gathered}
$$

where $\tilde{\nabla}$ denotes covariant differentiation in the connection $\tilde{\Gamma}$, and $\tilde{R}_{i j k}^{s}=\partial_{k} \tilde{\Gamma}_{i j}^{s}-\partial_{j} \tilde{\Gamma}_{i k}^{s}+$ $\tilde{\Gamma}_{i j}^{p} \tilde{\Gamma}_{p k}^{s}-\tilde{\Gamma}_{i k}^{p} \tilde{\Gamma}_{p j}^{s}$ is the curvature tensor. For our purposes, it will be more convenient to rewrite the compatibility conditions (2) in terms of the Levi-Civita connection $\Gamma_{i j}^{k}$ of the metric $g_{i j}$ and the difference $(1,2)$-tensor $h_{i j}^{k}$ defined by

$$
\tilde{\Gamma}_{i j}^{k}=\Gamma_{i j}^{k}+h_{i j}^{k}
$$

In this notation, equations (22) can be cast into the form [23]

$$
\begin{gathered}
h_{i j}^{s} g_{s k}=h_{i k}^{s} g_{s j}, \quad \nabla_{k} h_{i j}^{s}=\nabla_{j} h_{i k}^{s}, \\
R_{i j k}^{s}+h_{i j}^{p} h_{p k}^{s}-h_{i k}^{p} h_{p j}^{s}=g_{i k} \delta_{j}^{s}-g_{i j} \delta_{k}^{s}
\end{gathered}
$$

where $\nabla$ denotes covariant differentiation in the Levi-Civita connection $\Gamma$, and $R_{i j k}^{s}$ is the curvature tensor. Equation (3) ${ }_{1}$ implies that the tensor $h_{i j k}=h_{i j}^{s} g_{s k}$ is totally symmetric, defining the centroaffine cubic form of the hypersurface $M^{n}$. The centroaffine metric

$$
M=g_{i j} d u^{i} d u^{j}
$$

and the centroaffine cubic form

$$
C=h_{i j k} d u^{i} d u^{j} d u^{k}
$$

satisfying the compatibility conditions (3) characterize a hypersurface uniquely. Another useful invariant of a hypersurface in centroaffine geometry is the so-called Chebyshev covector,

$$
T=T_{i} d u^{i}=\frac{1}{n} h_{i s}^{s} d u^{i},
$$

which, as readily follows from (3) $)_{2}$, is always a gradient: $d T=0$. It is known that the vanishing of the cubic form characterizes central hyperquadrics [23]. The proper affine hyperspheres can be defined as hypersurfaces with the vanishing Chebyshev covector. The main object of our study is the third natural class of centroaffine hypersurfaces, namely, hypersurfaces with a flat centroaffine metric. Notice that the centroaffine metric can be equivalently defined by the formula

$$
M=\frac{\left|d^{2} \mathbf{r} \wedge \mathbf{r}_{1} \wedge \ldots \wedge \mathbf{r}_{n}\right|}{\left|\mathbf{r} \wedge \mathbf{r}_{1} \wedge \ldots \wedge \mathbf{r}_{n}\right|}
$$


For hypersurfaces defined explicitly as graphs of functions, $u^{n+1}=f\left(u^{1}, \ldots, u^{n}\right)$, the centroaffine metric has the form

$$
M=-\frac{d^{2} f}{F}=-\frac{d^{2} F}{F},
$$

where $F=f_{i} u^{i}-f$ is the Legendre transform of $f$, and $d^{2} F$ denotes the second differential. Some global aspects of Riemannian metrics of the form $-d^{2} F / F$ were discussed in 19 and [18]. The condition of flatness of the metric $-d^{2} F / F$ results in a system of third order nonlinear PDEs for the function $F$ (which looks quite formidable even in the simplest case $n=2$ ). As we demonstrate below, this system is nothing but the equations of associativity (WDVV equations).

Remark. System (11) appears naturally in the theory of multi-dimensional Schwarzian derivatives. In this setting, one is interested in the mapping from $M^{n}$ into the projective space $P^{n}$ defined as $\left(r^{0}: r^{1}: \ldots: r^{n}\right)$ in homogeneous coordinates of $P^{n}$. We refer to [26] for the details. In centroaffine geometry, non-constant rescalings of the position vector $\mathbf{r}$ are not allowed: they result in non-equivalent hypersurfaces.

\section{Hypersurfaces with flat centroaffine metric and associa- tivity equations}

For hypersurfaces with a flat centroaffine metric one can choose the parametrization such that $g_{i j}=\eta_{i j}=$ const, so that $\Gamma=0, \nabla_{k}=\partial_{k}$ and equations (3) take the form

$$
\begin{gathered}
h_{i j}^{s} \eta_{s k}=h_{i k}^{s} \eta_{s j}, \quad \partial_{k} h_{i j}^{s}=\partial_{j} h_{i k}^{s}, \\
h_{i j}^{p} h_{p k}^{s}-h_{i k}^{p} h_{p j}^{s}=\eta_{i k} \delta_{j}^{s}-\eta_{i j} \delta_{k}^{s} .
\end{gathered}
$$

The first two equations imply the existence of the potential $f$ such that

$$
h_{i j}^{s}=\eta^{s k} \partial_{i} \partial_{j} \partial_{k} f
$$

resulting in the following formulae for the centroaffine invariants:

centroaffine metric

$$
M=\eta_{i j} d u^{i} d u^{j}
$$

centroaffine cubic form

$$
C=d^{3} f
$$

Chebyshev covector

$$
T=\frac{1}{n} d(\triangle f)
$$

where $\triangle$ denotes the Laplacian in the metric $\eta_{i j}$.

In terms of $f$, the third set of equations (4) results in a nonlinear system of PDEs known as the equations of associativity in two-dimensional topological field theory [6]. To see this, we introduce one extra coordinate $u^{0}$ and the function

$$
F\left(u^{0}, u^{1}, \ldots, u^{n}\right)=\frac{1}{6}\left(u^{0}\right)^{3}+\eta_{i j} u^{0} u^{i} u^{j}+f\left(u^{1}, \ldots, u^{n}\right) .
$$


One can readily verify the following properties, which are in fact the axioms of the associativity equations (in what follows $\alpha, \beta, \gamma=0,1, \ldots, n$ and $i, j, k=1, \ldots, n$ ).

1. The matrix $\eta_{\alpha \beta}=\frac{\partial^{3} F}{\partial_{0} \partial_{\alpha} \partial_{\beta}}$ is constant and nondegenerate, indeed,

$$
\eta_{\alpha \beta}=\left(\begin{array}{cc}
1 & 0 \\
0 & \eta_{i j}
\end{array}\right)
$$

2. The objects $c_{\beta \gamma}^{\alpha}=\eta^{\alpha \delta} \frac{\partial^{3} F}{\partial_{\beta} \partial_{\gamma} \partial_{\delta}}$ are the structure constants of a commutative associative algebra. Indeed, the only nonzero among them are

$$
c_{00}^{0}=1, \quad c_{i j}^{0}=\eta_{i j}, \quad c_{0 j}^{i}=c_{j 0}^{i}=\delta_{j}^{i}, \quad c_{j k}^{i}=h_{j k}^{i},
$$

and equations (4) imply the associativity.

The function $F$ defines a Frobenius structure [6] on the manifold with coordinates $u^{0}, u^{1}, \ldots, u^{n}$, the main ingredients of which are the flat metric

$$
\left(d u^{0}\right)^{2}+\eta_{i j} d u^{i} d u^{j}
$$

and the symmetric 3 -tensor

$$
d^{3} F=\left(d u^{0}\right)^{3}+\eta_{i j} d u^{0} d u^{i} d u^{j}+d^{3} f .
$$

Notice that the centroaffine metric $M$ and the cubic form $C$ can be obtained by resticting these objects to a hyperplane $u^{0}=$ const in the spirit of [24]. Thus, hypersurfaces with flat centroaffine metric carry an intrinsic induced Frobenius structure. Moreover, one can incorporate the spectral parameter $\lambda$ into the equations for the position vector,

$$
\mathbf{r}_{i j}=\lambda h_{i j}^{k} \mathbf{r}_{k}+\lambda^{2} \eta_{i j} \mathbf{r}
$$

without violating the compatibility conditions (团). In arbitrary parametrization, the linear system (5) takes the form

$$
\mathbf{r}_{i j}=\left(\Gamma_{i j}^{k}+\lambda h_{i j}^{k}\right) \mathbf{r}_{k}+\lambda^{2} g_{i j} \mathbf{r}
$$

where $\Gamma$ is the Levi-Civita connection of the flat metric $g$. System (6) defines a oneparameter family of hypersurfaces $M_{\lambda}^{n}$ with centroaffine metrics

$$
M=\lambda^{2} g_{i j} d u^{i} d u^{j}
$$

and centroaffine cubic forms

$$
C=\lambda^{3} h_{i j k} d u^{i} d u^{j} d u^{k}
$$

Therefore, hypersurfaces with a flat centroaffine metric can be equivalently characterized as hypersurfaces possessing nontrivial deformations which induce rescalings of the centroaffine metric and the centroaffine cubic form. For $\lambda=1$ we recover the original hypersurface $M^{n}$, the case $\lambda=0$ corresponds to a hyperplane, and $\lambda=-1$ results in the dual hypersurface. 
Remark. One can consider the linear system (6) without imposing the restriction that $\Gamma$ is the Levi-Civita connection of $g$. In this case the compatibility conditions imply

$$
\begin{gathered}
\nabla_{k} g_{i j}=\nabla_{j} g_{i k}, \quad \nabla_{k} h_{i j}^{p}=\nabla_{j} h_{i k}^{p}, \quad h_{i j}^{s} g_{s k}=h_{i k}^{s} g_{s j} \\
h_{i j}^{p} h_{p k}^{s}-h_{i k}^{p} h_{p j}^{s}=g_{i k} \delta_{j}^{s}-g_{i j} \delta_{k}^{s}
\end{gathered}
$$

where $\nabla$ denotes covariant differentiation in the connection $\Gamma$. Moreover, the connection $\Gamma$ must be flat. This class of surfaces will be investigated in some more detail elsewhere.

Examples. In the case $n=2$ one can either choose $\eta=2 d x d y$ (hyperbolic surfaces) or $\eta=d x^{2}+d y^{2}$ (convex surfaces). Here $x=u^{1}$ and $y=u^{2}$ are asymptoticcoordinates on a surface $M^{2}$. In the hyperbolic case, equations (5) take the form

$$
\begin{gathered}
\mathbf{r}_{x x}=\lambda f_{x x x} \mathbf{r}_{y}+\lambda f_{x x y} \mathbf{r}_{x}, \\
\mathbf{r}_{x y}=\lambda f_{x x y} \mathbf{r}_{y}+\lambda f_{x y y} \mathbf{r}_{x}+\lambda^{2} \mathbf{r}, \\
\mathbf{r}_{y y}=\lambda f_{x y y} \mathbf{r}_{y}+\lambda f_{y y y} \mathbf{r}_{x},
\end{gathered}
$$

with the compatibility condition

$$
f_{x x x} f_{y y y}-f_{x x y} f_{y y x}=1 .
$$

In a similar form (however, without a spectral parameter) the linear problem (8) was presented in [7]. Introducing $\psi=\left(\lambda \mathbf{r}, \mathbf{r}_{x}, \mathbf{r}_{y}\right)^{T}$, one can readily rewrite (8) in a matrix form

$$
\psi_{x}=\lambda\left(\begin{array}{ccc}
0 & 1 & 0 \\
0 & f_{x x y} & f_{x x x} \\
1 & f_{x y y} & f_{x x y}
\end{array}\right) \psi, \quad \psi_{y}=\lambda\left(\begin{array}{ccc}
0 & 0 & 1 \\
1 & f_{x y y} & f_{x x y} \\
0 & f_{y y y} & f_{x y y}
\end{array}\right) \psi
$$

which coincides with the one from [6]. Similarly, in the convex case we have

$$
\begin{gathered}
\mathbf{r}_{x x}=\lambda f_{x x x} \mathbf{r}_{x}+\lambda f_{x x y} \mathbf{r}_{y}+\lambda^{2} \mathbf{r}, \\
\mathbf{r}_{x y}=\lambda f_{x x y} \mathbf{r}_{x}+\lambda f_{x y y} \mathbf{r}_{y}, \\
\mathbf{r}_{y y}=\lambda f_{x y y} \mathbf{r}_{x}+\lambda f_{y y y} \mathbf{r}_{y}+\lambda^{2} \mathbf{r},
\end{gathered}
$$

with the compatibility condition

$$
f_{x x y}^{2}+f_{x y y}^{2}-f_{x x x} f_{x y y}-f_{y y y} f_{x x y}=1 .
$$

Particular examples of surfaces with a flat centroaffine metric were discussed in 22 (surfaces of revolution with the centroaffine metric of constant curvature) and [17] (centroaffinely flat Chebyshev surfaces).

Introducing the variables $f_{x x x}=p, f_{x x y}=a, f_{x y y}=b$ and $f_{y y y}=q$, [20], [8], one can rewrite (9) in the form

$$
p_{y}=a_{x}, \quad a_{y}=b_{x}, \quad b_{y}=q_{x}, \quad p q-a b=1
$$


which is equivalent to the quasilinear system

$$
\left(\begin{array}{l}
p \\
a \\
b
\end{array}\right)_{y}=U\left(\begin{array}{l}
p \\
a \\
b
\end{array}\right)_{x}, \quad U=\left(\begin{array}{ccc}
0 & 1 & 0 \\
0 & 0 & 1 \\
-\frac{1+a b}{p^{2}} & \frac{b}{p} & \frac{a}{p}
\end{array}\right)
$$

investigated in detail in $[8]$. The characteristics of this system are defined by $d x+\lambda d y=0$ where $\lambda$ is a root of the characteristic polynomial of the matrix $U$,

$$
p \lambda^{3}-a \lambda^{2}-b \lambda+q=0 .
$$

Equivalently, characteristics can be defined as the null-curves of the cubic form

$$
p d x^{3}+a d x^{2} d y-b d x d y^{2}-q d y^{3},
$$

indeed, $\lambda=-d x / d y$. The geometry of this cubic form is clarified in section 4 .

\section{Surfaces in centroaffine 3-space}

In asymptotic coordinates $x$ and $y$ (for definiteness, we consider hyperbolic case only), equations (1) take the form

$$
\begin{aligned}
& \mathbf{r}_{x x}=\left(a+u_{x}\right) \mathbf{r}_{x}+p \mathbf{r}_{y}, \\
& \mathbf{r}_{x y}=b \mathbf{r}_{x}+a \mathbf{r}_{y}+e^{u} \mathbf{r}, \\
& \mathbf{r}_{y y}=q \mathbf{r}_{x}+\left(b+u_{y}\right) \mathbf{r}_{y},
\end{aligned}
$$

with the compatibility conditions

$$
\begin{gathered}
a_{y}=b_{x}, \quad a_{x}-p_{y}=a u_{x}+p u_{y}, \quad b_{y}-q_{x}=q u_{x}+b u_{y}, \\
p q-a b=e^{u}(K+1),
\end{gathered}
$$

where $K=-e^{-u} u_{x y}$ is the Gaussian curvature of the corresponding centroaffine metric

$$
M=2 e^{u} d x d y
$$

Notice that in the flat case $(K=0, u=0)$ equations (16) coincide with (13). Since the only nonzero Christoffel symbols of the centroaffine metric $M$ are $\Gamma_{11}^{1}=u_{x}$ and $\Gamma_{22}^{2}=u_{y}$, we have $h_{11}^{1}=a, h_{11}^{2}=p, h_{12}^{1}=b, h_{12}^{2}=a, h_{22}^{1}=q$ and $h_{22}^{2}=b$, so that the centroaffine cubic form $C$ and the Chebyshev covector $T$ take the forms

$$
C=e^{u}\left(p d x^{3}+3 a d x^{2} d y+3 b d x d y^{2}+q d y^{3}\right)
$$

and

$$
T=a d x+b d y
$$

respectively. 
Remark. We point out that surfaces with the zero Chebyshev covector are the proper affine spheres, indeed, the substitution of $a=b=0$ into $(16))_{2}$ and $(\sqrt{16})_{3}$ implies $p=q=$ $e^{-u}$, so that $(16)_{4}$ results in the Tzitzeica equation $u_{x y}=e^{u}-e^{-2 u}$.

Let us consider the form

$$
C-2 T M=e^{u}\left(p d x^{3}-a d x^{2} d y-b d x d y^{2}+q d y^{3}\right)
$$

which, at a generic point of our surface, has three distinct zero directions. The three directions conjugate to them are defined by the equation

$$
p d x^{3}+a d x^{2} d y-b d x d y^{2}-q d y^{3}=0,
$$

(recall that two directions are called conjugate if they are orthogonal with respect to the metric $M)$. The null-curves of (17) define a foliation of our surface by three families of curves, which we will call the characteristic 3-web for the reason which is clarified at the end of section 3 .

Theorem 1 For surfaces with a flat centroaffine metric, the characteristic 3-web is hexagonal.

\section{Proof:}

A direct calculation of the curvature form $\Omega$ of the characteristic 3 -web results in the formula $\Omega=d \omega$, where the connection form $\omega$ is

$$
\omega=-\frac{3 e^{u}}{r^{2}-4 s t}\left(\left(r K_{x}-2 t K_{y}\right) d x+\left(r K_{y}-2 s K_{x}\right) d y\right),
$$

here $r=a b-9 p q, s=b^{2}+3 a q, t=a^{2}+3 b p$. If $K=0$, the curvature form $\Omega$ is zero, implying the hexagonality of the characteristic 3 -web.

Remark. The class of surfaces with a hexagonal characteristic 3 -web includes surfaces with a flat centroaffine metric as a proper subclass. For instance, the form of $\omega$ implies that surfaces with the centroaffine metric of constant curvature ( $K=$ const) also have a hexagonal characteristic web. In would be of interest to discuss this class of surfaces in some more detail. The simplest examples of surfaces with centroaffine metric of constant curvature $K=-1$ are central quadrics (which correspond to $a=b=p=q=0$ in the equations (16)). Since in this case (17) is identically zero, the characteristic 3 -web is indeterminate.

\section{Other parametrizations}

In asymptotic coordinates $x$ and $y$, the position vector $\mathbf{r}$ of a surface with flat centroaffine metric satisfies the equations

$$
\begin{gathered}
\mathbf{r}_{x x}=\lambda a \mathbf{r}_{x}+\lambda p \mathbf{r}_{y} \\
\mathbf{r}_{x y}=\lambda b \mathbf{r}_{x}+\lambda a \mathbf{r}_{y}+\lambda^{2} \mathbf{r} \\
\mathbf{r}_{y y}=\lambda q \mathbf{r}_{x}+\lambda b \mathbf{r}_{y}
\end{gathered}
$$


the compatibility conditions of which coincide with (13). After the reparametrization $(x, y) \rightarrow(t, y)$ where $t$ is defined by

$$
d t=p d x+a d y
$$

equations (18) take the form

$$
\begin{gathered}
\mathbf{r}_{t t}=\left(\frac{C_{t}}{C}-2 \lambda B\right) \mathbf{r}_{t}+\lambda C \mathbf{r}_{y} \\
\mathbf{r}_{t y}=\left(\frac{B_{t}}{C}-\lambda A\right) \mathbf{r}_{t}+\lambda^{2} C \mathbf{r} \\
\mathbf{r}_{y y}=\left(\lambda+\frac{B_{y}}{C}\right) \mathbf{r}_{t}-\lambda A \mathbf{r}_{y}+2 \lambda^{2} B \mathbf{r},
\end{gathered}
$$

where we have introduced the notation

$$
A=a^{2} / p-b, \quad B=-a / p, \quad C=1 / p .
$$

The compatibility conditions of equations (20) take the form

$$
A_{t}=B_{y}, \quad B_{t}=C_{y}, \quad C_{t}=\left(B^{2}-A C\right)_{y} .
$$

Written in terms of the potential $F$ such that $A=F_{y y y}, B=F_{t y y}$ and $C=F_{t t y}$, this system reduces to a single $\mathrm{PDE}$

$$
F_{t t t}=F_{t y y}^{2}-F_{t t y} F_{y y y}
$$

which is yet another form of the associativity equations [6]. Thus, both equations (9) and (22) describe one in the same class of surfaces, however, in different parametrizations. Notice that, in terms of the potentials $f(x, y)$ and $F(x, t)$, the formulae (19) and (21) take the form

$$
t=f_{x x}, \quad F_{y y y}=\frac{f_{x x y}^{2}}{f_{x x x}}-f_{x y y}, \quad F_{t y y}=-\frac{f_{x x y}}{f_{x x x}}, \quad F_{t t y}=\frac{1}{f_{x x x}}, \quad F_{t t t}=\frac{f_{x y y}}{f_{x x x}},
$$

and, after being integrated once, simplify to

$$
f_{x x}=t, \quad f_{x y}=-F_{y y}, \quad f_{y y}=F_{t t}, \quad x=F_{t y} .
$$

This transformation between (9) and (22) was first proposed in [8]. Taking, for instance, the known polynomial solution of the equation (22),

$$
F(t, y)=\frac{t^{2} y^{2}}{4}+\frac{t^{5}}{60}
$$

we readily arrive at the formulae

$$
f_{x x}=t, \quad f_{x y}=-\frac{t^{2}}{2}, \quad f_{y y}=\frac{y^{2}}{2}+\frac{t^{3}}{3}, \quad x=t y,
$$

which, after the substitution of $t=x / y$ into the first three equations, give a solution of (9):

$$
f(x, y)=\frac{x^{3}}{6 y}+\frac{y^{4}}{24}
$$


Here we list some further solutions of the equation $f_{x x x} f_{y y y}-f_{x x y} f_{y y x}=1$ which can be obtained by applying the transformation (23) to the known solutions of (22) as found in [6]:

\begin{tabular}{|l|l|}
\hline$F_{t t t}=F_{t y y}^{2}-F_{t t y} F_{y y y}$ & $f_{x x x} f_{y y y}-f_{x x y} f_{y y x}=1$ \\
\hline$F=\frac{t^{2} y^{2}}{4}+\frac{t^{5}}{60}$ & $f=\frac{x^{3}}{6 y}+\frac{y^{4}}{24}$ \\
\hline$F=y e^{t}-\frac{y^{4}}{24}$ & $f=\frac{x y^{3}}{6}+\frac{x^{2} \ln x}{2}-\frac{3 x^{2}}{4}$ \\
\hline$F=\frac{y^{2} e^{t}}{4}+\frac{e^{2 t}}{32}-\frac{y^{4}}{48}$ & $f=\frac{x y^{3}}{12}-\frac{x^{2} \ln y}{2}+\frac{x^{2} \ln x}{2}-\frac{3 x^{2}}{4}$ \\
\hline$F=\frac{t^{2} \ln y}{2}$ & $f=\frac{y x^{3}}{6}+\frac{y^{2} \ln y}{2}-\frac{3 y^{2}}{4}$ \\
\hline$F=\frac{y^{3} t}{6}+\frac{y^{2} t^{3}}{6}+\frac{t^{7}}{210}$ & $f=\frac{4 y^{2}}{15}\left(\frac{x}{y}-\frac{y}{2}\right)^{5 / 2}$ \\
\hline$F=\frac{y^{3} t^{2}}{6}+\frac{y^{2} t^{5}}{20}+\frac{t^{11}}{3960}$ & $f=\frac{y^{4} t^{3}}{6}+\frac{7 y^{3} t^{6}}{30}+\frac{4 y^{2} t^{9}}{45}+\frac{y^{5}}{60}, \quad$ where $x=y^{2} t+\frac{y t^{4}}{2}$ \\
\hline
\end{tabular}

Notice that the second and fourth solutions in the right column differ by the interchange of $x$ and $y$, which is an obvious symmetry of the corresponding PDE. The last solution is implicit. The geometry of the corresponding surfaces is currently under investigation.

The characteristic parametrization. Introducing the variables $w^{1}, w^{2}, w^{3}$ by the formulae

$$
w^{1}+w^{2}+w^{3}=2 a, \quad w^{1} w^{2}+w^{1} w^{3}+w^{2} w^{3}=a^{2}-p b, \quad w^{1} w^{2} w^{3}=p,
$$

one can rewrite equations (13) in a symmetric form [8],

$$
w_{y}^{1}=\left(\frac{w^{1}-w^{2}-w^{3}}{2 w^{2} w^{3}}\right)_{x}, \quad w_{y}^{2}=\left(\frac{w^{2}-w^{1}-w^{3}}{2 w^{1} w^{3}}\right)_{x}, \quad w_{y}^{3}=\left(\frac{w^{3}-w^{1}-w^{2}}{2 w^{1} w^{2}}\right)_{x} .
$$

This system possesses three characteristic conservation laws

$$
\begin{gathered}
\left(w^{2}-w^{3}\right)\left(d x+\frac{w^{1}+w^{2}-w^{3}}{2 w^{1} w^{2} w^{3}} d y\right), \quad\left(w^{1}-w^{3}\right)\left(d x+\frac{w^{1}+w^{3}-w^{2}}{2 w^{1} w^{2} w^{3}} d y\right) \\
\left(w^{2}-w^{1}\right)\left(d x+\frac{w^{2}+w^{3}-w^{1}}{2 w^{1} w^{2} w^{3}} d y\right)
\end{gathered}
$$

(we call a conservation law 'characteristic' if its null curves are characteristics of the system (24)). After introducing the characteristic coordinates $\xi$ and $\eta$,

$$
\begin{gathered}
d \xi=\left(w^{2}-w^{3}\right)\left(d x+\frac{w^{1}+w^{2}-w^{3}}{2 w^{1} w^{2} w^{3}} d y\right), \quad d \eta=\left(w^{1}-w^{3}\right)\left(d x+\frac{w^{1}+w^{3}-w^{2}}{2 w^{1} w^{2} w^{3}} d y\right), \\
d \xi-d \eta=\left(w^{2}-w^{1}\right)\left(d x+\frac{w^{2}+w^{3}-w^{1}}{2 w^{1} w^{2} w^{3}} d y\right)
\end{gathered}
$$


equations (18) for the position vector take the form

$$
\begin{aligned}
& \mathbf{r}_{\xi \xi}=\left(\Gamma_{11}^{1}+\lambda h_{11}^{1}\right) \mathbf{r}_{\xi}+\left(\Gamma_{11}^{2}+\lambda h_{11}^{2}\right) \mathbf{r}_{\eta}+\lambda^{2} E \mathbf{r}, \\
& \mathbf{r}_{\xi \eta}=\left(\Gamma_{12}^{1}+\lambda h_{12}^{1}\right) \mathbf{r}_{\xi}+\left(\Gamma_{12}^{2}+\lambda h_{12}^{2}\right) \mathbf{r}_{\eta}+\lambda^{2} F \mathbf{r}, \\
& \mathbf{r}_{\eta \eta}=\left(\Gamma_{22}^{1}+\lambda h_{22}^{1}\right) \mathbf{r}_{\xi}+\left(\Gamma_{22}^{2}+\lambda h_{22}^{2}\right) \mathbf{r}_{\eta}+\lambda^{2} G \mathbf{r},
\end{aligned}
$$

where the coefficients of the centroaffine metric $M=E d \xi^{2}+2 F d \xi d \eta+G d \eta^{2}$ are given by

$$
\begin{gathered}
E=\frac{w^{1} w^{2} w^{3}\left(w^{2}-w^{1}-w^{3}\right)}{\left(w^{2}-w^{1}\right)^{2}\left(w^{2}-w^{3}\right)^{2}}, \quad F=\frac{w^{1} w^{2}\left(w^{3}\right)^{2}}{\left(w^{1}-w^{2}\right)^{2}\left(w^{1}-w^{3}\right)\left(w^{2}-w^{3}\right)}, \\
G=\frac{w^{1} w^{2} w^{3}\left(w^{1}-w^{2}-w^{3}\right)}{\left(w^{1}-w^{2}\right)^{2}\left(w^{1}-w^{3}\right)^{2}}
\end{gathered}
$$

and $\Gamma_{j k}^{i}$ are the components of the corresponding Levi-Civita connection. The coefficients $h_{j k}^{i}$ are of the form

$$
\begin{gathered}
h_{11}^{1}=\frac{\left(w^{2}\right)^{2}-w^{1} w^{2}-w^{1} w^{3}-w^{2} w^{3}}{\left(w^{2}-w^{1}\right)\left(w^{2}-w^{3}\right)}, \quad h_{22}^{2}=\frac{\left(w^{1}\right)^{2}-w^{1} w^{2}-w^{1} w^{3}-w^{2} w^{3}}{\left(w^{1}-w^{2}\right)\left(w^{1}-w^{3}\right)}, \\
h_{12}^{1}=-\frac{w^{2} w^{3}}{\left(w^{1}-w^{2}\right)\left(w^{1}-w^{3}\right)}, \quad h_{11}^{2}=h_{22}^{1}=0, \quad h_{12}^{2}=-\frac{w^{1} w^{3}}{\left(w^{2}-w^{1}\right)\left(w^{2}-w^{3}\right)} .
\end{gathered}
$$

We point out that the components $E, F$ and $G$ of the flat metric $M$ satisfy the identity

$$
\left(E G-F^{2}\right)^{2}+F(E+F)(G+F)=0 .
$$

In coordinates $\xi$ and $\eta$, the characteristic 3 -web is defined by the equation $d \xi d \eta(d \xi-d \eta)=$ 0 , and is manifestly hexagonal. The compatibility conditions of equations (25) take the form

$$
\begin{gathered}
\frac{w^{3}-w^{2}}{w^{1}} w_{\xi}^{1}=\frac{w^{3}-w^{1}}{w^{2}} w_{\eta}^{2}, \\
\frac{w^{2}-w^{3}}{w^{1}}\left(w_{\xi}^{1}+w_{\eta}^{1}\right)=\frac{w^{1}-w^{2}}{w^{3}} w_{\eta}^{3}, \\
\frac{w^{1}-w^{3}}{w^{2}}\left(w_{\xi}^{2}+w_{\eta}^{2}\right)=\frac{w^{2}-w^{1}}{w^{3}} w_{\xi}^{3},
\end{gathered}
$$

and, upon the introduction of variables $u^{i}=1 / w^{i}$, simplify to

$$
\begin{gathered}
\left(u^{3}-u^{2}\right) u_{\xi}^{1}=\left(u^{3}-u^{1}\right) u_{\eta}^{2}, \\
\left(u^{2}-u^{3}\right)\left(u_{\xi}^{1}+u_{\eta}^{1}\right)=\left(u^{1}-u^{2}\right) u_{\eta}^{3}, \\
\left(u^{1}-u^{3}\right)\left(u_{\xi}^{2}+u_{\eta}^{2}\right)=\left(u^{2}-u^{1}\right) u_{\xi}^{3} .
\end{gathered}
$$

System (26) can be represented in the equivalent exterior form

$$
\omega^{1} \wedge d \xi=0, \quad \omega^{2} \wedge d \eta=0, \quad \omega^{3} \wedge(d \xi-d \eta)=0,
$$


with the 1 -forms

$$
\begin{aligned}
& \omega^{1}=\frac{\left(u^{2}-u^{3}\right) d u^{1}+\left(u^{1}-u^{3}\right) d u^{2}+\left(u^{2}-u^{1}\right) d u^{3}}{2\left(u^{2}-u^{3}\right) \sqrt{\left(u^{2}-u^{1}\right)\left(u^{3}-u^{1}\right)}}, \\
& \omega^{2}=\frac{\left(u^{2}-u^{3}\right) d u^{1}+\left(u^{1}-u^{3}\right) d u^{2}+\left(u^{1}-u^{2}\right) d u^{3}}{2\left(u^{3}-u^{1}\right) \sqrt{\left(u^{2}-u^{1}\right)\left(u^{2}-u^{3}\right)}}, \\
& \omega^{3}=\frac{\left(u^{2}-u^{3}\right) d u^{1}+\left(u^{3}-u^{1}\right) d u^{2}+\left(u^{2}-u^{1}\right) d u^{3}}{2\left(u^{2}-u^{1}\right) \sqrt{\left(u^{3}-u^{1}\right)\left(u^{2}-u^{3}\right)}}
\end{aligned}
$$

satisfying the structure equations of the $S O(2,1)$ group,

$$
d \omega^{1}=\omega^{2} \wedge \omega^{3}, \quad d \omega^{2}=\omega^{3} \wedge \omega^{1}, \quad d \omega^{3}=\omega^{2} \wedge \omega^{1} .
$$

Therefore, one can set

$$
\omega^{1}=p^{1} d \xi, \quad \omega^{2}=p^{2} d \eta, \quad \omega^{3}=p^{3}(d \xi-d \eta),
$$

and the substitution into (27) implies the 3 -wave system

$$
p_{\eta}^{1}=p^{2} p^{3}, \quad p_{\xi}^{2}=p^{1} p^{3}, \quad p_{\xi}^{3}+p_{\eta}^{3}=p^{1} p^{2} .
$$

It should be pointed out that the equivalence of the associativity equations and the Nwave system was first observed by Dubrovin [6]. The explicit sequence of transformations shown above (which maps (9) into (28)) was presented in [8], see also [1].

\section{$6 \quad$ Examples}

Particular exact solutions of the system (13) correspond to special surfaces with a flat centroaffine metric.

Example 1. Solutions of system (14) with a degenerate hodograph (rarefaction waves) are of the form

$p=\varphi(x+c y), \quad a=c \varphi(x+c y)+\mu, \quad b=c^{2} \varphi(x+c y)-\frac{1}{\mu}, \quad q=c^{3} \varphi(x+c y)+c^{2} \mu-\frac{c}{\mu}$,

where $c$ and $\mu$ are arbitrary constants and $\varphi$ is an arbitrary function of $x+c y$. The corresponding potential $f$ is

$$
f(x, y)=\psi(x+c y)+\frac{\mu}{2} x^{2} y-\frac{1}{2 \mu} x y^{2}+\frac{1}{6}\left(c^{2} \mu-c / \mu\right) y^{3},
$$

here $\psi^{\prime \prime \prime}=\varphi$. The corresponding equations (18) (where we have set $\lambda=1$ ) take the form

$$
\begin{gathered}
\mathbf{r}_{x x}=(c \varphi+\mu) \mathbf{r}_{x}+\varphi \mathbf{r}_{y} \\
\mathbf{r}_{x y}=\left(c^{2} \varphi-\frac{1}{\mu}\right) \mathbf{r}_{x}+(c \varphi+\mu) \mathbf{r}_{y}+\mathbf{r}, \\
\mathbf{r}_{y y}=\left(c^{3} \varphi+c^{2} \mu-\frac{c}{\mu}\right) \mathbf{r}_{x}+\left(c^{2} \varphi-\frac{1}{\mu}\right) \mathbf{r}_{y}
\end{gathered}
$$


In the new parametrization $t=(x+c y) / 2, s=(x-c y) / 2$, these equations can be rewritten as

$$
\begin{gathered}
\mathbf{r}_{s s}=\left(2 \mu+\frac{1}{c \mu}\right) \mathbf{r}_{s}-\frac{2}{c} \mathbf{r} \\
\mathbf{r}_{s t}=\frac{1}{c \mu} \mathbf{r}_{t} \\
\mathbf{r}_{t t}=\left(4 c \varphi+2 \mu-\frac{2}{c \mu}\right) \mathbf{r}_{t}-\frac{1}{c \mu} \mathbf{r}_{s}+\frac{2}{c} \mathbf{r}
\end{gathered}
$$

implying

$$
\left(\mathbf{r}-c \mu \mathbf{r}_{s}\right)_{s}=2 \mu\left(\mathbf{r}-c \mu \mathbf{r}_{s}\right), \quad\left(\mathbf{r}-c \mu \mathbf{r}_{s}\right)_{t}=0
$$

Therefore,

$$
\mathbf{r}=e^{2 \mu s} \mathbf{R}_{\mathbf{0}}+e^{\frac{s}{c \mu}} \mathbf{R}(t)
$$

where $\mathbf{R}_{\mathbf{0}}$ is a constant vector and $\mathbf{R}(t)$ satisfies the ODE

$$
\mathbf{R}^{\prime \prime}=\left(4 c \varphi+2 \mu-\frac{2}{c \mu}\right) \mathbf{R}^{\prime}+\left(\frac{2}{c}-\frac{1}{c^{2} \mu^{2}}\right) \mathbf{R} .
$$

Hence, $\mathbf{R}(t)$ is a planar curve (which can be arbitrary since $\varphi$ is an arbitrary function of $t$ ), and the intersection of our surface with the plane spanned by $\mathbf{R}_{\mathbf{0}}$ and $\mathbf{R}$ has parametric equation $\left(e^{2 \mu s}, e^{\frac{s}{c \mu}}\right)$.

Example 2. One can show that, in appropriate asymptotic parametrization, surfaces of revolution with a flat centroaffine metric correspond to the solution

$$
a=y \alpha(\xi), \quad b=x \beta(\xi), \quad p=y^{3} \rho(\xi), \quad q=x^{3} \gamma(\xi)
$$

where $\xi=x y$ and the functions $\alpha, \beta, \rho, \gamma$ satisfy the ODEs

$$
\alpha^{\prime}=3 \rho+\xi \rho^{\prime}, \quad \alpha+\xi \alpha^{\prime}=\beta+\xi \beta^{\prime}, \quad \beta^{\prime}=3 \gamma+\xi \gamma^{\prime}, \quad \xi^{3} \rho \gamma-\xi \alpha \beta=1 .
$$

The general solution of this system can be parametrized in the form

$$
\alpha=2 G+\xi G^{\prime}, \quad \beta=2 H+\xi H^{\prime}, \quad \rho=G^{\prime}, \quad \gamma=H^{\prime}
$$

where the functions $G(\xi)$ and $H(\xi)$ solve the quadratic system

$$
\xi^{2}(H-G)=\epsilon \xi+\nu, \quad 2 \xi^{2} H G=\mu-\xi,
$$

$\epsilon, \nu, \mu$ being arbitrary constants. In a different parametrization, these surfaces were also discussed in [22]. Equivalently, the above solutions can be characterized in terms of the corresponding potential

$$
f(x, y)=F(\xi)+\left(s_{1} \xi+s_{2}\right) \ln x+\left(r_{1} \xi+r_{2}\right) \ln y,
$$

where $\xi=x y$ and $s_{1}, s_{2}, r_{1}, r_{2}$ are arbitrary constants. Calculation of the third order derivatives of $f$ gives

$$
\begin{gathered}
a=f_{x x y}=y\left(\xi F^{\prime \prime \prime}+2 F^{\prime \prime}+\frac{s_{1}}{\xi}\right), \quad b=f_{x y y}=x\left(\xi F^{\prime \prime \prime}+2 F^{\prime \prime}+\frac{r_{1}}{\xi}\right), \\
p=f_{x x x}=y^{3}\left(F^{\prime \prime \prime}-\frac{s_{1}}{\xi^{2}}+2 \frac{s_{2}}{\xi^{3}}\right), \quad q=f_{y y y}=x^{3}\left(F^{\prime \prime \prime}-\frac{r_{1}}{\xi^{2}}+2 \frac{r_{2}}{\xi^{3}}\right)
\end{gathered}
$$


(so that $H=F^{\prime \prime}+r_{1} / \xi-r_{2} / \xi^{2}, G=F^{\prime \prime}+s_{1} / \xi-s_{2} / \xi^{2}$ ), and the substitution into (9) implies the quadratic equation for $F^{\prime \prime}$,

$$
\xi^{2}\left(F^{\prime \prime}\right)^{2}+\left(r_{1}+s_{1}\right) \xi F^{\prime \prime}-\left(r_{2}+s_{2}\right) F^{\prime \prime}-\frac{r_{1} s_{2}+r_{2} s_{1}}{\xi}+\frac{r_{2} s_{2}}{\xi^{2}}+\frac{1}{2} \xi+s=0,
$$

where $s$ is a constant of integration.

Some further explicit solutions of equation (9) can be sought in the form

$$
f=y^{3 / 2} F(x),
$$

where $F$ satisfies the $\operatorname{ODE}\left(F^{2}\right)^{\prime \prime \prime}=-16 / 3$, implying $F(x)=\sqrt{-\frac{8}{9} x^{3}+\alpha x^{2}+\beta x+\gamma}$, $\alpha, \beta, \gamma$ being arbitrary constants. Another possible choice is

$$
f=y^{3} F(\xi), \quad \xi=x / y,
$$

implying the following third order ODE for $F$,

$$
6 F F^{\prime \prime \prime}-4 \xi F^{\prime} F^{\prime \prime \prime}+2 \xi\left(F^{\prime \prime}\right)^{2}-2 F^{\prime} F^{\prime \prime}=1 .
$$

A more general self-similar substitution is

$$
f=(x y)^{3 / 2} F\left(x y^{\mu}\right)
$$

where $\mu=$ const. For $\mu=0$ and $\mu=-1$ this reduces to (30) and (31), respectively.

\section{The loop group formulation of surfaces with a flat cen- troaffine metric}

The results of this section are due to A. Bobenko. Substituting $\psi=e^{\frac{2}{3} \lambda f_{x y}} \varphi$ into (10), we obtain the equivalent traceless representation

$$
\varphi_{x}=\lambda\left(\begin{array}{ccc}
-\frac{2}{3} a & 1 & 0 \\
0 & \frac{1}{3} a & p \\
1 & b & \frac{1}{3} a
\end{array}\right) \varphi, \quad \varphi_{y}=\lambda\left(\begin{array}{ccc}
-\frac{2}{3} b & 0 & 1 \\
1 & \frac{1}{3} b & a \\
0 & q & \frac{1}{3} b
\end{array}\right) \varphi
$$

where we have set $f_{x x x}=p, f_{x x y}=a, f_{x y y}=b, f_{y y y}=q$. Let us introduce the loop group of $3 \times 3$ matrices

$$
G=\left\{\varphi(\lambda) \mid T\left(\varphi^{t}(-\lambda)\right)^{-1} T=\varphi(\lambda), \operatorname{det} \varphi(\lambda)=1\right\}, \quad T=\left(\begin{array}{ccc}
1 & 0 & 0 \\
0 & 0 & 1 \\
0 & 1 & 0
\end{array}\right),
$$

with the corresponding loop algebra

$$
g=\left\{A(\lambda) \mid-T A^{t}(-\lambda) T=A(\lambda), \operatorname{tr} A(\lambda)=0\right\} .
$$


Lemma. Let $\varphi(t, \lambda) \rightarrow G$ be such that $\varphi_{t} \varphi^{-1}$ is linear in $\lambda$, and $\left.\varphi_{t} \varphi^{-1}\right|_{\lambda=0}=0$. Then

$$
\varphi_{t} \varphi^{-1}(t, \lambda)=\lambda\left(\begin{array}{ccc}
-2 A & C & D \\
D & A & P \\
C & B & A
\end{array}\right) .
$$

This readily implies the following

Proposition. Let $\varphi(u, v, \lambda) \rightarrow G$ be an immersion of an open domain of $R^{2}$ such that $d \varphi \varphi^{-1}$ is linear in $\lambda$, and $\left.d \varphi \varphi^{-1}\right|_{\lambda=0}=0$. Then there exists a change of variables $(u, v) \rightarrow(x, y)$ bringing the frame equations into the form (32).

\section{Projective generalizations of hypersurfaces with a flat cen- troaffine metric}

There exist two particularly interesting projectively invariant classes of hypersurfaces containing projective transforms of hypersurfaces with flat centroaffine metric.

Hypersurfaces with conformally flat second fundamental form were investigated in [1], [2] and [15] (notice that this property makes sense only for hypersurface of dimension greater than two). Among previosly known examples one should mention the second order envelopes of one-parameter families of nondegenerate hyperquadrics (in particular, projective transforms of surfaces of revolution) as well as special projections of the Segre variety $P^{2} \times P^{2} \subset P^{8}$ into $P^{5}$. We point out that projective transforms of hypersurfaces with flat centroaffine metric provide further examples of this type. Indeed, the conformal class of the second fundamental form of a hypersurface with flat centroaffine metric is $\eta_{i j} d u^{i} d u^{j}$, which is manifestly conformally flat.

Surfaces in $P^{3}$ possessing asymptotic deformations which induce rescalings of the projective metric and the Darboux cubic form. Let us recall that the position vector $\mathbf{R}$ of a surface $M^{2}$ in the projective space $P^{3}$ satisfies the linear system

$$
\begin{aligned}
& \mathbf{R}_{x x}=\beta \mathbf{R}_{y}+\frac{1}{2}\left(V-\beta_{y}\right) \mathbf{R} \\
& \mathbf{R}_{y y}=\gamma \mathbf{R}_{x}+\frac{1}{2}\left(W-\gamma_{x}\right) \mathbf{R}
\end{aligned}
$$

where $\beta, \gamma, V, W$ are functions of the asymptotic coordinates $x$ and $y$ satisfying the compatibility conditions [16, p. 120]

$$
\begin{gathered}
\beta_{y y y}-2 \beta_{y} W-\beta W_{y}=\gamma_{x x x}-2 \gamma_{x} V-\gamma V_{x} \\
W_{x}=2 \gamma \beta_{y}+\beta \gamma_{y} \\
V_{y}=2 \beta \gamma_{x}+\gamma \beta_{x}
\end{gathered}
$$

The most important projective invariants are the projective metric

$$
2 \beta \gamma d x d y
$$


and the conformal class of the Darboux cubic form

$$
\beta d x^{3}+\gamma d y^{3}
$$

Paticularly interesting classes of projective surfaces are characterized by additional relations among $\beta, \gamma, V$ and $W$, for instance

- isothermally-asymptotic surfaces $(\beta=\gamma)$;

- projectively applicable surfaces $\left(\beta_{y}=\gamma_{x}\right)$;

- surfaces of Jonas $\left(\beta_{x}=\gamma_{y}\right)$;

etc (see [3], [16], [10]).

Here we consider another class of projective surfaces characterised by the additional relation

$$
\beta_{y y y}=\gamma_{x x x}
$$

which allows one to introduce the spectral parameter $\lambda$ into (33):

$$
\begin{gathered}
\mathbf{R}_{x x}=\lambda \beta \mathbf{R}_{y}+\frac{1}{2}\left(\lambda^{2} V-\lambda \beta_{y}\right) \mathbf{R} \\
\mathbf{R}_{y y}=\lambda \gamma \mathbf{R}_{x}+\frac{1}{2}\left(\lambda^{2} W-\lambda \gamma_{x}\right) \mathbf{R} .
\end{gathered}
$$

Since the projective metric and the conformal class of the cubic form of the one-parameter family of surfaces $M_{\lambda}^{2}$ defined by $(\overline{36})$ are

$$
2 \lambda^{2} \beta \gamma d x d y
$$

and

$$
\beta d x^{3}+\gamma d y^{3},
$$

respectively, one can say that the surfaces satisfying (35) possess one-parameter families of asymptotic deformations which rescale the projective metric and preserve the conformal class of the Darboux cubic form. As $\lambda$ varies, we continuosly change from the initial surface $(\lambda=1)$ to a quadric $(\lambda=0)$, and then to the dual surface $(\lambda=-1)$. Surfaces satisfying the condition (35) were discussed in [5] and [14]. Changing in the equations (8) from $\mathbf{r}$ to $\mathbf{R}=e^{-\frac{1}{2} \lambda f_{x y}} \mathbf{r}$, we readily rewrite (8) 1 and $(8)_{3}$ in the form (36), where

$$
\beta=f_{x x x}, \quad \gamma=f_{y y y}, \quad V=\frac{1}{2} f_{x x y}^{2}+f_{x x x} f_{x y y}, \quad W=\frac{1}{2} f_{x y y}^{2}+f_{y y y} f_{x x y}
$$

and $f$ satisfies the associativity equation (9). Therefore, projective transforms of surfaces (8) form a proper subclass of the integrable class of surfaces characterized by the condition (35).

\section{Acknowledgements}

It is a great pleasure to thank A. I. Bobenko for numerous discussions and for proving the material presented in sect. 7. This research was partially supported by SFB 288 and the EPSRC grant GR/N30941. 


\section{References}

[1] Akivis M.A. and Goldberg V.V., Conformal differential geometry and its generalizations, John Wiley and Sons, 1996.

[2] Akivis M.A. and Konnov V.V., Local aspects in conformal structure theory, Russian Math. Surveys 48, no. 1 (1993) 1-35.

[3] Bol G., Projektive Differentialgeometrie, Göttingen, 1954.

[4] Cartan E., Sur la déformation projective des surfaces, Ann. de l'Ecole Normale Sup., (3) 37 (1920) 259-356.

[5] Cech E., Sur les correspondances asymptotiques entre deux surfaces, Rendiconti Accademia Nazionale dei Lincei, Roma 6 ser. 8 (1928) 484-486; 552-554.

[6] Dubrovin B. A., Geometry of 2D topological field theories, Lecture Notes in Mathematics, V.1620, Berlin, Springer, 120-348.

[7] Dryuma V., On the Riemannian and Einstein-Weyl geometry in the theory of second order ordinary differential equations, arXiv:math.DG/0104278.

[8] Ferapontov E. V. and Mokhov O. I., Equations of associativity of two-dimensional topological field theory as integrable Hamiltonian nondiagonalisable systems of hydrodynamic type, Funkt. Anal. and it's Appl., 30, N3 (1996) 62-72.

[9] Ferapontov E. V. and Schief W. K., Surfaces of Demoulin: Differential geometry, Bäcklund transformation and Integrability, Journal of Geometry and Physics 30 (1999) 343-363.

[10] Ferapontov E.V. Integrable systems in projective differential geometry, Kyushu J. Math. 54 (2000) 183-215.

[11] Ferapontov E. V., Galvao C. A. P., Mokhov O. I. and Nutku Y. , Bi-Hamiltonian structure of equations of associativity in 2-d topological field theory, Comm. in Math. Phys., 186 (1997) 649-669.

[12] Finikov S. P., Projective Differential Geometry, Moscow-Leningrad, 1937.

[13] Fubini G. and Ĉech E., Geometria Proiettiva Differenziale, Bologna: Zanichelli, 1926.

[14] Kaucký J., Sur les transformations asymptotiques d'une surface non dévelopable en elle même dans l'espace projectif $S_{3}$, Mém. Bohême 16 (1932) 1-35.

[15] Konnov V.V., Asymptotic pseudoconformal structure on a four-dimensional hypersurface and its completely isotropic two-dimensional submanifolds, Russian Math. 36, no. 6 (1992) 67-74.

[16] Lane E., Treatise on Projective Differential Geometry, The Univ. of Chicago Press, 1942. 
[17] Liu H. and Wang C., The centroaffine Chebyshev operator, Results in Math. 27 (1995) 77-92.

[18] Loewner C. and Nirenberg L., Partial differential equations invariant under conformal or projective transformations, in Contributions to Analysis, Academic Press (1974) $245-272$.

[19] Loftin J. C., Riemannian metrics on locally projectively flat manifolds, arXiv:math.DG/0108218

[20] Mokhov O.I., Symplectic and Poisson geometry on loop spaces of manifolds and nonlinear equations, AMS Translations ser. 2170 (1995).

[21] Sasaki T., Projective differential geometry and linear homogeneous differential equations, Rokko lectures in Math., Kobe University, Japan, 5 (1999) 1-115, ISBN 4907719-05-1.

[22] Scharlach C., Some results in centroaffine differential geometry, in: Geometry and Topology of Submanifolds, 4, ed. F. Dillen and L. Verstraelen, World Scientific, Singapore, (1992) 198-206.

[23] Shirokov P. A. and Shirokov A. P., Affine Differential geometry, Moscow, 1959.

[24] Strachan I.A.B., Frobenius submanifolds, J. Geom. Phys. 38 (2001) 285-307.

[25] Wilczynski E. I., Projective-differential geometry of curved surfaces, Trans. AMS 8 (1907) 233-260; 9 (1908) 79-120, 293-315.

[26] Yoshida M., Fuchsian Differential Equations, Vieweg, 1987. 